\title{
Research Paper \\ Relationship of Perceived Social Support With the Activities of Daily Living in Older Adults Living in Rural Communities in Iran
}

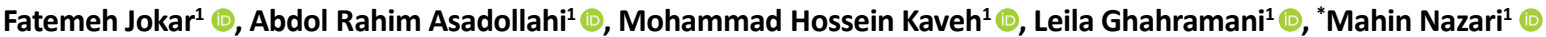

1. Department of Health Education and Health Promotion, School of Health, Shiraz University of Medical Sciences, Shiraz, Iran.

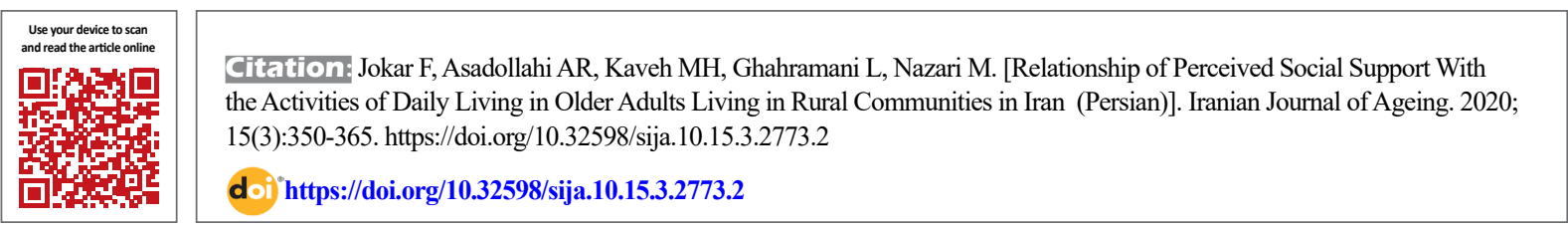

\section{(i) $(5)$}

Received: 09 Nov 2019

Accepted: 17 May 2020

Available Online: 01 Oct 2020
Key words:

Old people, Rural older adults, Social support, Activities of daily living

\section{A B STRACT}

Objectives The increasing trend in aging population raises the need to pay attention to the daily activities of the elderly and their social support as an effective factor in promoting their health. This study aims to investigate the correlation of perceived social support and demographic variables with the Activities of Daily Living (ADL) in older adults living in rural communities in Iran.

Methods \& Materials This is a descriptive-analytical study with cross-sectional design conducted on 430 elderly people aged $\leq 60$ years living in a rural community in Iran (Bayza county located 45 km away from Shiraz city) who were selected using a convenience sampling method. Procidano \& Heller's Perceived Social Support - Family Scale (PSS-Fa) and the ADL scale for the elderly were used for data collection. The ADL questionnaire's internal consistency by calculating Cronbach's alpha coefficient was obtained 0.96. The PSS-Fa with a Cronbach's alpha coefficient ranging from 0.88 to 0.91 has acceptable internal consistency. The reliability of its Persian version using Cronbach's alpha coefficient was obtained $87 \%$. Data were analyzed in SPSS V. 25 software using multivariate ANOVA and regression analysis.

Results The Mean \pm SD age of the participants was $69.67 \pm 7.067$ years. The Mean score of PSS-Fa and the ADL scale was reported $16.55 \pm 5.16$ and 55.10 \pm 3.07 , respectively. Perceived social support, education and age had significant effect correlation with the ability to perform $A D L$ in the elderly $(P<0.001)$, while marital status and gender showed no significant correlation $(P>0.05)$. The age factor was inversely correlated with the ability to perform ADL; hence, the independence of older adults decreases with the increase of age. Conclusion Many demographic variables and social support affect the ability to perform ADL in the elderly. Social support can be used as a social investment to improve the quality of life of the elderly. Therefore, considering that one of the duties of health care providers is to improve the health status of the elderly, it is necessary for health care providers to increase social support and maintain and promote a healthy and active life for them through educational programs and periodic physical examinations.

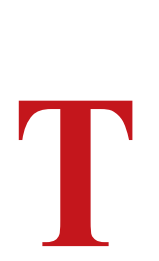

\section{Extended Abstract}

\section{Introduction}

he growing trend in aging population raises the need to pay attention to the daily activities of the elderly and their social support as an effective factor in promoting their health. Although friendship and support are concepts as old as human communication, the concept of social support is a relatively new concept. Today, diseases and injuries are studied in correlation with social support. The present study aims to investigate the relationship of perceived social support and demo-

"Corresponding Author:

Mahin Nazari, PhD.

Address: Department of Health Education and Health Promotion, School of Health, Shiraz University of Medical Sciences, Shiraz, Iran.

Tel: +98 (71) 32305001

E-mail: manazari@sums.ac.ir 
graphic variables with Activities of Daily Living (ADLs) in the elderly live in rural communities.

\section{Methods \& Materials}

This is a descriptive/analytical study. The study population consists of the elderly live in Bayza County located 45 kilometers away from the city of Shiraz, Iran. This county has 6 districts, and one district (Harabal) that was easier to access, was selected. This district has 8 health centers. The present study was conducted in the villages of Haft Khan, Haji Abad, Aliabad Tang, Ayazjan, Jafarabad, Ebrahimabad. The sample size was determined 400 according to the previous study [32] and using the formula, which increased to 420 by considering $\alpha=0.05$, test power $(\beta-1)=$ $80 \%$ and a drop rate of $20 \%$. Samples were selected using a convenience sampling method. Inclusion criteria were: hearing and speaking abilities, age 60 or older, having an active medical record in comprehensive health databases, and declaring informed consent. Exclusion criteria were: return of incomplete questionnaire, unwillingness to cooperate, and the presence of diagnosed diseases. Data collection tools were Procidano \& Heller's Perceived Social Support - Family Scale (PSS-Fa) and the ADL scale for the elderly. The internal consistency of the ADL questionnaire using Cronbach's alpha coefficient in Iranian samples was calculated 0.96. The PSS-Fa has a Cronbach's alpha coefficient of 0.88-0.91. For its Persian version, a Cronbach's alpha coefficient of 0.87 was obtained. Data analysis was performed in SPSS V. 25 software using multivariate ANO$\mathrm{VA}$ and regression analysis.

\section{Results}

The Mean \pm SD age of participants was $69.67 \pm 7.067 ; 60.2 \%$ aged $60-70$ years, $33 \%$ aged $71-80$ years, and $6.8 \%$ aged 80 years and older. Moreover, $51.6 \%$ were women and the rest were men; $79.3 \%$ were married and $20.5 \%$ were widowed;

Table 1. Multivariate ANOVA Results of comparing the effect of perceived social support on the ADLs of older adults in rural areas

\begin{tabular}{|c|c|c|c|c|c|c|c|c|}
\hline Variable & & Source & Sum of Squares & df & Mean Square & $\mathbf{F}$ & Sig. & Partial Eta Squared \\
\hline \multirow{18}{*}{$\begin{array}{l}\text { Perceived social } \\
\text { support }\end{array}$} & & Between-group & 4860.508 & 17 & 2850.370 & & & \\
\hline & $\begin{array}{l}\text { Heavy work } \\
\text { activities }\end{array}$ & Within-group & 36409.308 & 412 & 700.628 & 3.235 & 0.000 & 0.118 \\
\hline & & Total & 41269.816 & 429 & & & & \\
\hline & & Between-group & 2204.952 & 17 & 285.912 & & & \\
\hline & $\begin{array}{l}\text { Religious } \\
\text { activities }\end{array}$ & Within-group & 25939.515 & 412 & 88.372 & 2.060 & 0.008 & 0.178 \\
\hline & & Total & 28144.467 & 429 & & & & \\
\hline & & Between-group & 1413.027 & 17 & 129.703 & & & \\
\hline & $\begin{array}{l}\text { Personal } \\
\text { care }\end{array}$ & Within-group & 14984.138 & 412 & 62.960 & 1.863 & 0.003 & 0.186 \\
\hline & & Total & 16397.165 & 429 & & & & \\
\hline & & Between-group & 1183.530 & 17 & 119.83 & & & \\
\hline & $\begin{array}{c}\text { Subtle } \\
\text { activities }\end{array}$ & Within-group & 7363.121 & 412 & 36.369 & 2.285 & 0.000 & 0.138 \\
\hline & & Total & 8546.651 & 429 & & & & \\
\hline & & Between-group & 406.847 & 17 & 69.619 & & & \\
\hline & Washing & Within-group & 2362.150 & 382 & 17.872 & 3.235 & & 0.147 \\
\hline & & Total & 2768.998 & 399 & & & & \\
\hline & & Between-group & 48456.258 & 17 & 23.932 & & & \\
\hline & Overall & Within-group & 252926.796 & 361 & 184.6 & 068.4 & 0.000 & 0.248 \\
\hline & & Total & 301383.082 & 378 & 2850.370 & & & \\
\hline
\end{tabular}


$80.2 \%$ were illiterate and the rest had primary education. The mean score of perceived social support was obtained $16.55 \pm 5.16$ (ranging 0-20) and the mean score of ADL scale was $55.10 \pm 3.7$ (ranging 0-90), indicating relatively high social support and independence of the elderly. 59.21\% of the elderly with the age of $60-70$ years, $51.93 \%$ with $80-71$ years, and $41.24 \%$ with the age of 81 years and older were able to perform daily activities, indicating that with increasing age, the daily activities of the elderly decrease.

Multivariate ANOVA Results reported ICC $=0.979(95 \%)$ CI:0./975 (95\%), and showed that the factors of marital status and gender had no significant effect on the ADL of the elderly in rural areas $(\mathrm{P}>0.05)$. According to Table 1 , multivariate ANOVA Results showed that perceived social support had a significant effect on the overall ADL and its subscales (subtle activities, washing and heavy work activities). For heavy work activities, religious activities, personal care, subtle activities and the overall ADL, Eta Squared coefficient were $0.118,0.178,0.186,0.138,0.147$, and 0.248 , respectively. This indicates that social support can explain $24.8 \%$ of the total ADL of the elderly in rural areas, and high social support increases the ADLs of the elderly (Adjusted $\mathrm{R}^{2}=0.288, \mathrm{P} \leq 0.05$ ).

\section{Conclusion}

Many demographic variables and social support affect the ADL level of the elderly. Social support can be used as a social investment to improve the quality of life of the elderly. Therefore, considering that one of the duties of health care providers is to improve the health status of the elderly, it is necessary for health care providers to increase social support and maintain and promote a healthy and active life for them through educational programs and periodic physical examinations.

\section{Ethical Considerations}

Compliance with ethical guidelines

This study obtained its ethical approval from the Research Ethics Committee of Shiraz University of Medical Sciences (Code: SUMS.11128). Informed consent was obtained from all participants prior to sudy.

Funding

This study received financial support from the Deputy for Research of Shiraz University of Medical Sciences.

\section{Authors' contributions}

Conceptualization, interview: Fatemeh Jokar; Supervision and writing - original draft: Mahin Nazari; Methodology and data analysis: Abdolrahim Asadollahi; Approve: A ll authors.

\section{Conflicts of interest}

The authors declare no conflict of interest

\section{Acknowledgements}

The authors would like to thank the older adults participated in the study for their valuable cooperation. 


\title{
ارتباط بين حمايت اجتماعى و كيفيت فعاليت روزمره سالمندان جامعه روستايى
}

فاطمه جوكار' ه. عبدالرحيم اسداللهى'، محمد حسين كاوه'، ليلا قهرمانى '،"مهين نظرى'

ا. كروه آموزش بهداشت و ارتقاى سلامت، دانشكده بهداشت، دانشكاه علوميز شكى شيراز، شيرازء ايران.

\begin{abstract}
حكs

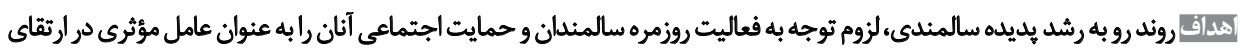

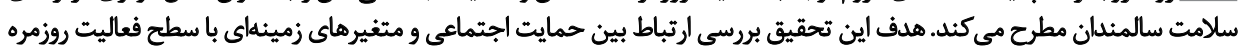

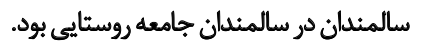

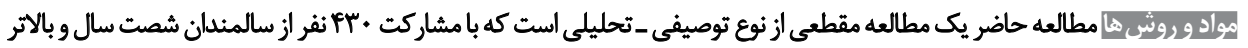

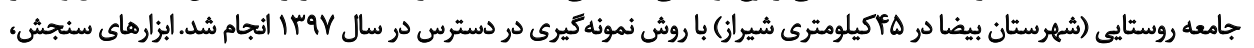

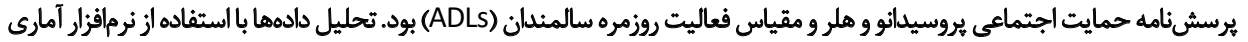

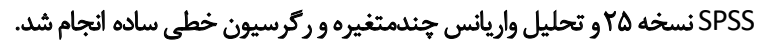

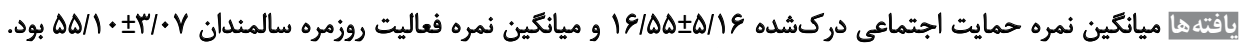

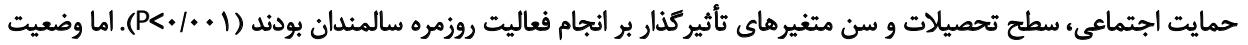

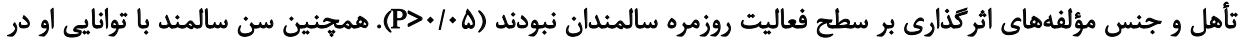

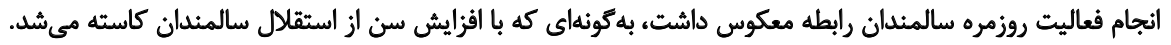

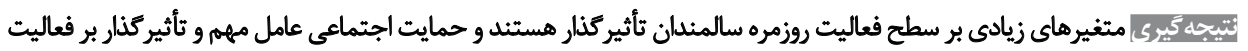

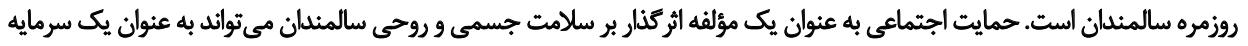

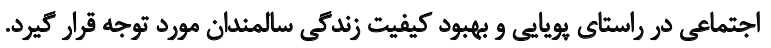

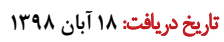

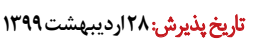
تاريخ انتشار: 11 تير 149

$$
\begin{aligned}
& \text { : } 100 \text { glguls } \\
& \text { سالمندان روسثايي، } \\
& \text { فعاليت روزمره } \\
& \text { سالمندان، حمايت } \\
& \text { اجتماعى سالينان }
\end{aligned}
$$

قدرت عضلانى، انعطافيذيرى محدود و كاهش توده استخوانى

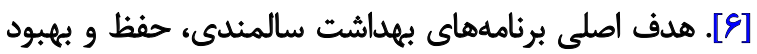

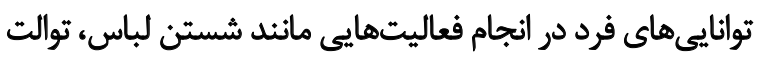

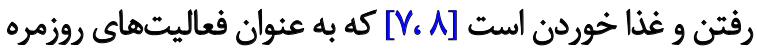

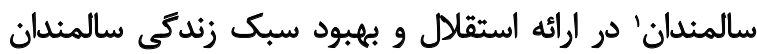

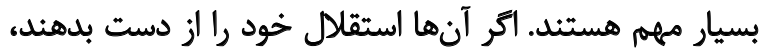

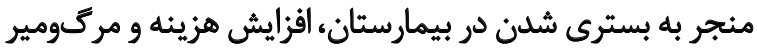

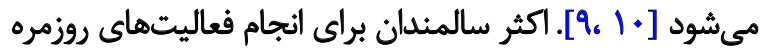

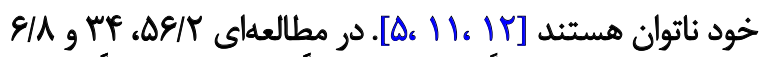

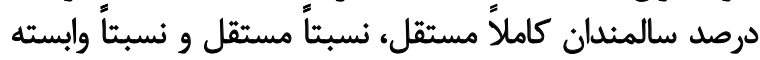

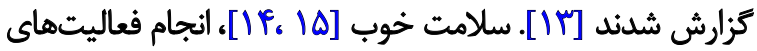

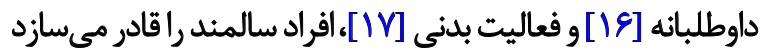

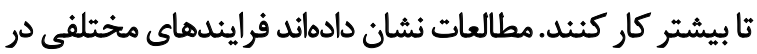

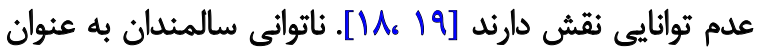

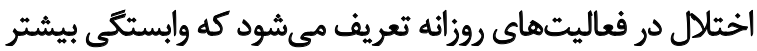

1. Activities of Daily Living (ADLs)

مقدمه

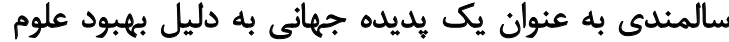

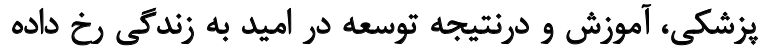

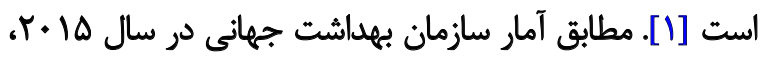

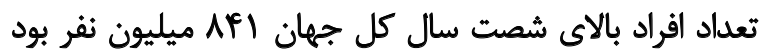

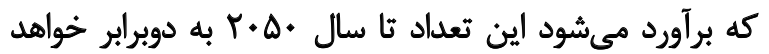

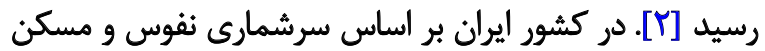

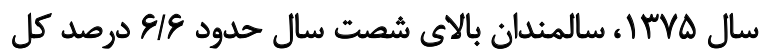

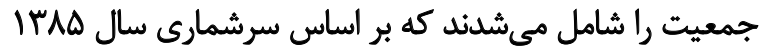

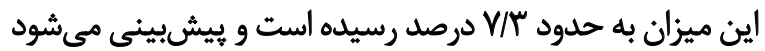

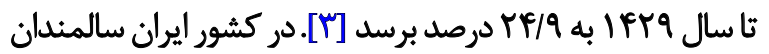

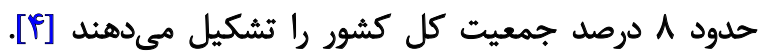

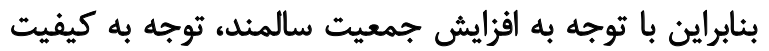

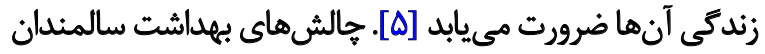

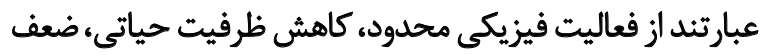

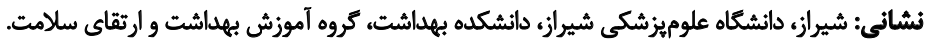

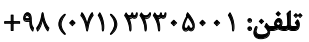
بـ 
در روستاهاى هفتخوان، حاجي آباد، على آباد تنك، ايازجان،

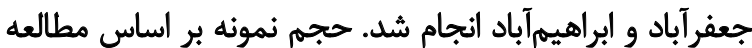

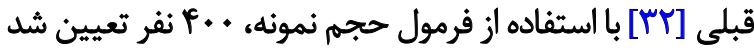

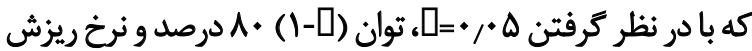

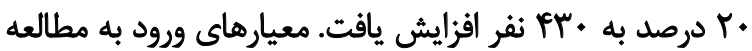

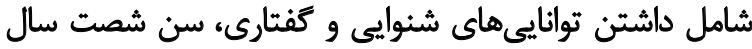

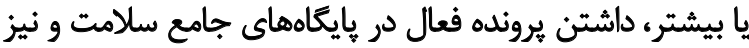

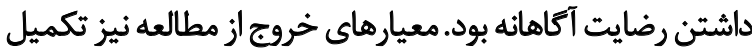

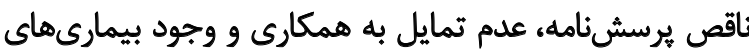
تشخيص دادشده در مطالعه در نظر كرفته شد.

ابزار جمعآورى دادهها شامل دو برسشنامه فعاليت روزمره

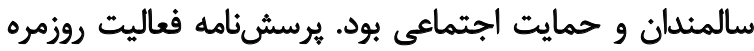

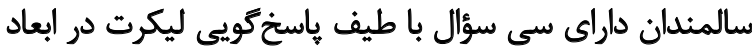

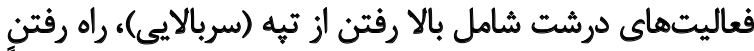

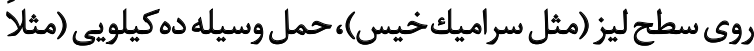

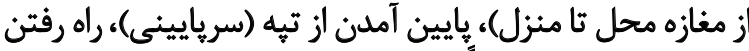

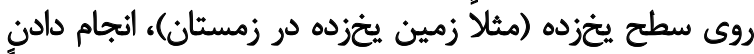

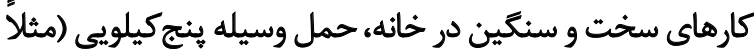

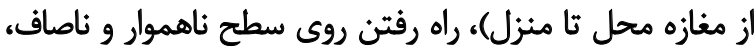

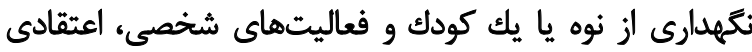

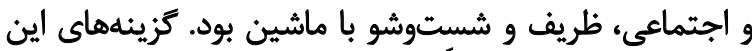

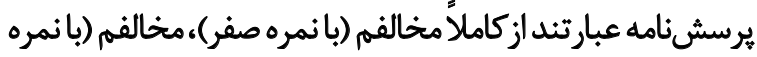

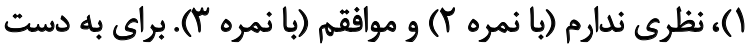

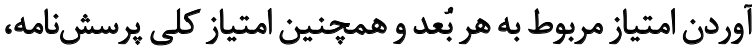

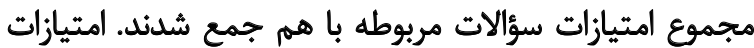

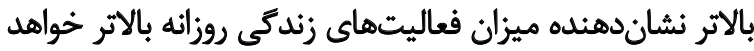

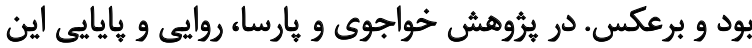

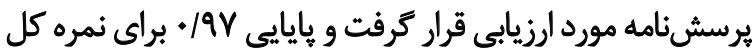

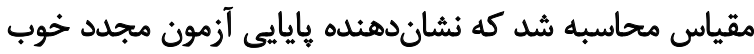

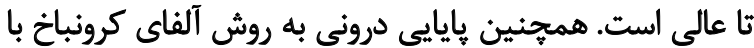

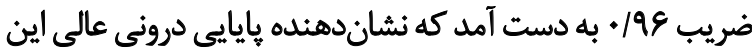

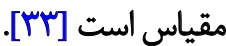

برسش نامه حمايت اجتماعى (مقياس خانواده) كه توسط مرى

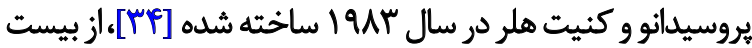

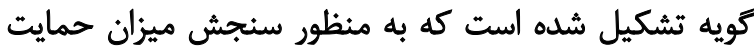

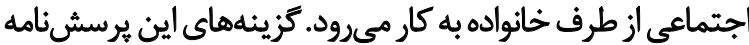

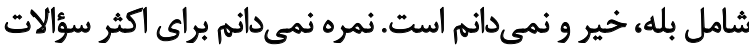

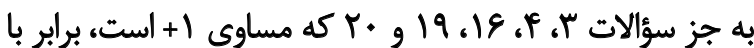

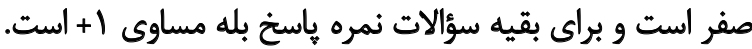

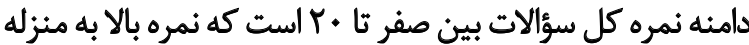

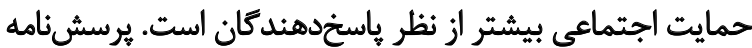

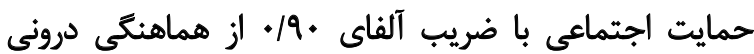

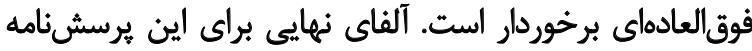

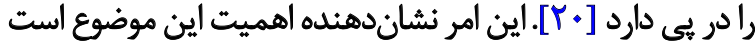

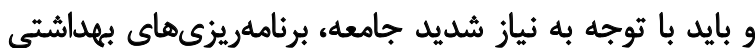
درمانى مناسب براي اين گروه سنى تهيه و اجرا شودي حمايت اجتماعى عامل مهمى در حفظ و ارتقاى سلامت

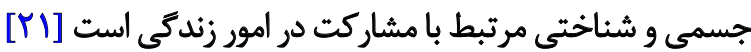

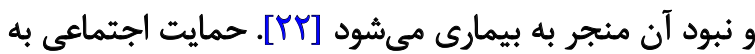

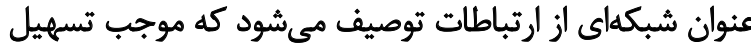

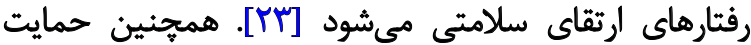

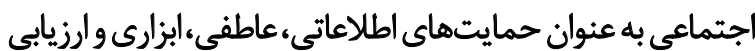

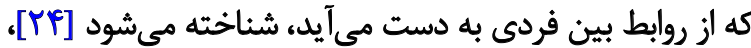

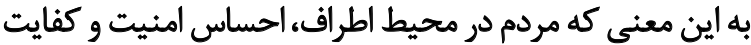

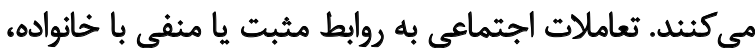

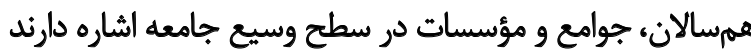

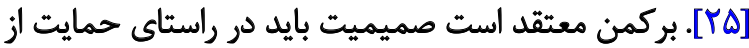

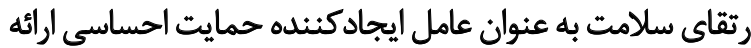

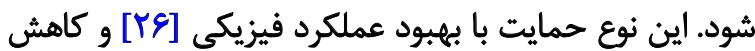

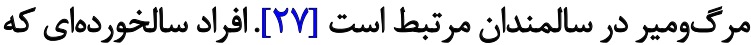

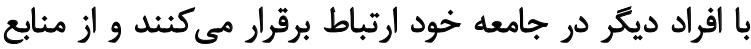

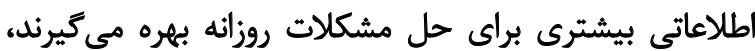

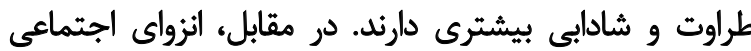

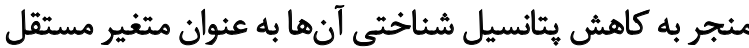

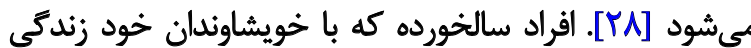

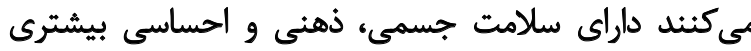

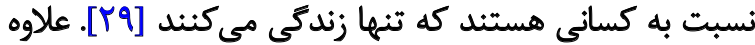

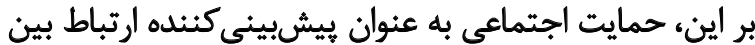

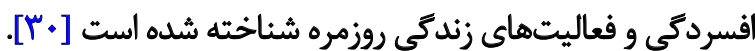

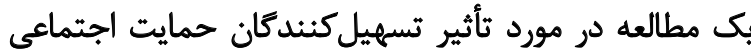

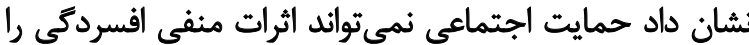

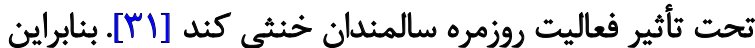

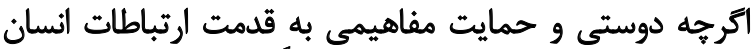

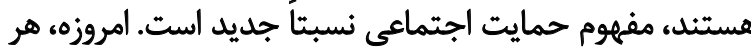

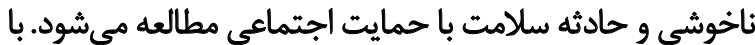

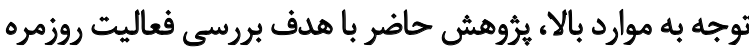

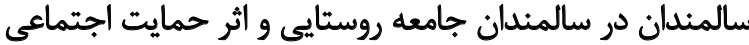
دركشده و متغيرهاى زمينهاى بر آن انجام شد.

$$
\text { روش مطالعه }
$$

يرؤش حاضر يك مطالعه توصيفى - تحليلى است كه در

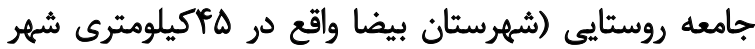

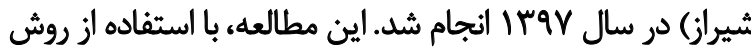

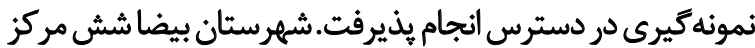

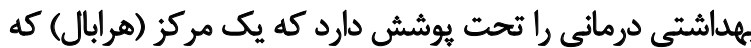

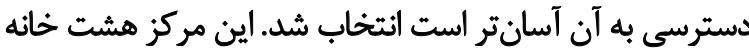

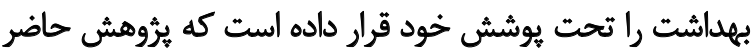




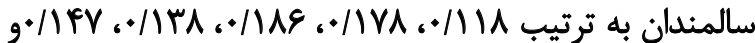

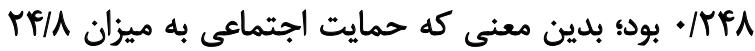

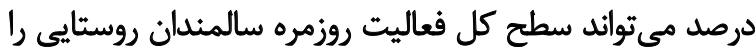

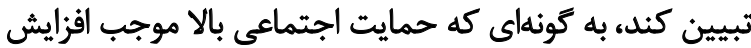

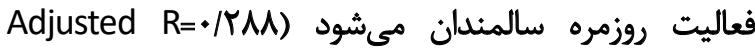
. $(P<+. \Delta$, Square

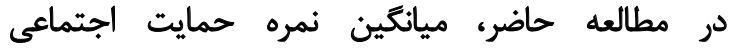

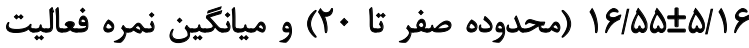

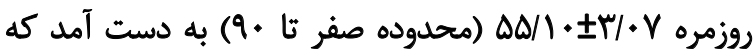

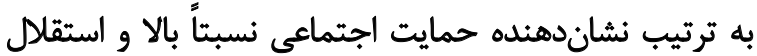
سالمندان است (جدول شماره \&).

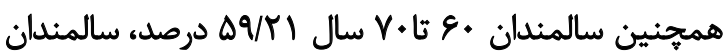

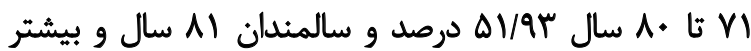
إزيش FI/TF

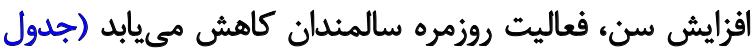

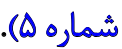

\section{بحث}

در مطالعه حاضر،ميانكين نمرهحمايت اجتماعى

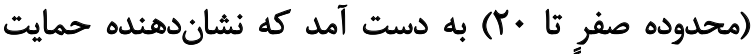

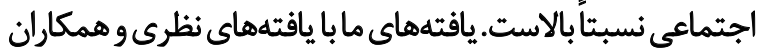

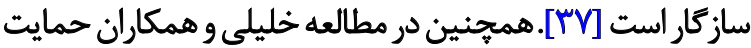

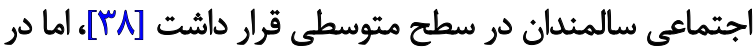

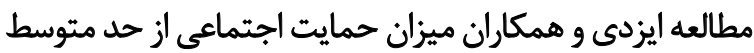

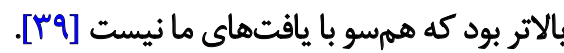

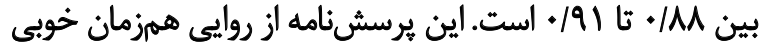

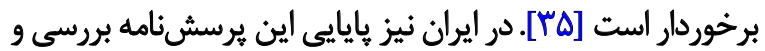

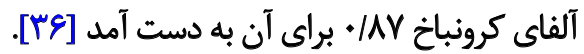

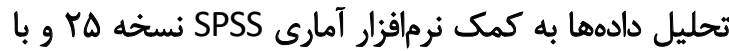

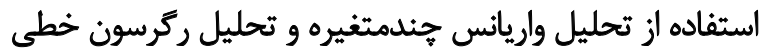

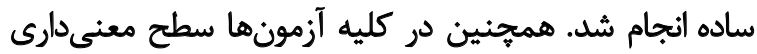

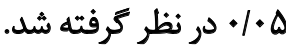

ياقتهذها

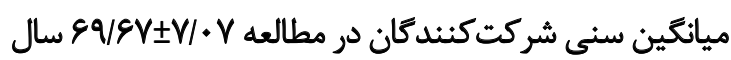

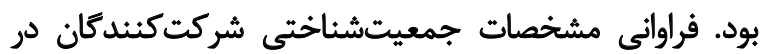
جدول شماره آورده شده است.

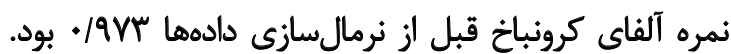

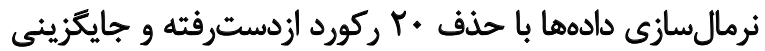

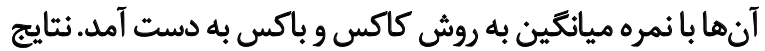

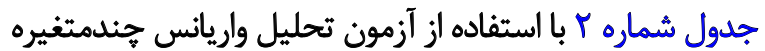

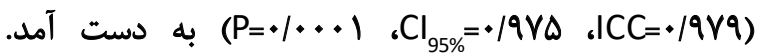
تحليل واريانس هندمتغيره نشان داد مؤلفهاهاى وضعيت تأهل و جنسيت اثر معنى دارى بر سطح فعاليت روزمره سالمندان ندارند . $(P>\cdot / \cdot \Delta)$

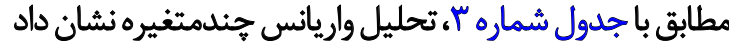

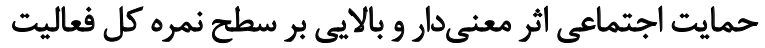

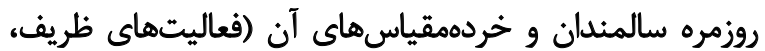

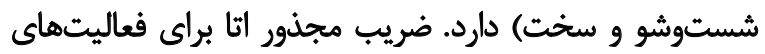
سخت،اعتقادى، شخصى، ظريف و سطح نمره كل فعاليت روزمره

جدول ا. مشخصات جمعيتشناختى سالمندان منطقه روستايى شُهرستان بيضا (•FT نفر )

\begin{tabular}{|c|c|c|}
\hline تعداد (درصد) & طبقدبندى & متغيرها \\
\hline$r \cdot \Lambda(F N F)$ & مرد & \multirow[b]{2}{*}{ جنس } \\
\hline $\operatorname{MT}(\Delta / / 9)$ & زن & \\
\hline $1(\cdot / \pi)$ & مجرد & \multirow{3}{*}{ وضعيت تأهل } \\
\hline mil (va/r) & متأهل & \\
\hline$M(r \cdot \Delta)$ & بيوه & \\
\hline $\operatorname{Tes}(\lambda . / \pi)$ & بىسواد & \multirow{2}{*}{ ميزان تحصيلات } \\
\hline$\Lambda \Delta(19 / \Lambda)$ & ابتدايي & \\
\hline$r \Delta q(\xi+\pi)$ & ع عتا V مال & \multirow{3}{*}{ رددبندى سنى } \\
\hline $\operatorname{IPT}(\pi T / \cdot)$ & 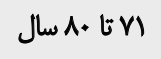 & \\
\hline$r q(g / V)$ & اN سال و ييشتر & \\
\hline
\end{tabular}

$\therefore$ 


\begin{tabular}{|c|c|c|c|c|c|c|c|c|}
\hline ضريب مجذور اتا & $\mathbf{P}$ & $\mathbf{F}$ & ميانكين مربعات & دوجه آزادى & مجموع مربعاث & اريانس & 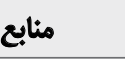 & مثغير اثر كَذار \\
\hline \multirow{3}{*}{.$/ 109$} & \multirow{3}{*}{$+1+\infty$} & \multirow{3}{*}{$T / \Delta) \Delta$} & TINWE & $r$ & EACT/TVA & بين كروهى & \multirow{3}{*}{ فغاليت } & \multirow{18}{*}{ سن } \\
\hline & & & Ne/UF & rq9 & $M F V+g / \Delta H^{\prime} A$ & درون كروهى & & \\
\hline & & & $r+\Delta / v \varepsilon$ & req & FIrEq/AIE & كل & & \\
\hline \multirow{3}{*}{.$/ M T$} & \multirow{3}{*}{$.1 \ldots$} & \multirow{3}{*}{$r / . \Delta q$} & ITA/YeA & $r$ & $T W T / \Gamma \Delta$. & يين كروهي & \multirow{3}{*}{ اعتقادى } & \\
\hline & & & $8 V \cdot A r$ & rq9 & METVIIIV & درون كروهي & & \\
\hline & & & WEIAYA & prq & rulfieler & كل & & \\
\hline \multirow{3}{*}{. } & \multirow{3}{*}{$.1 .+4$} & \multirow{3}{*}{ WNEY } & EV/\QV & $r$ & r.IF/N. & بين Sروهي & \multirow{6}{*}{ ظريف } & \\
\hline & & & rel.pe & rqq & IFTAYTFPA & درون كروهي & & \\
\hline & & & $1 \cdot r / r \cdot r$ & prq & IETYVIIFA & كل & & \\
\hline \multirow{3}{*}{.1 .94} & \multirow{3}{*}{.$N \cdot Y$} & \multirow{3}{*}{$1 / T \Delta q$} & $r e / f+1$ & $r$ & V9r/rro & بين كروهى & & \\
\hline & & & 19/4Ta & 199 & War/Fis & درون كروهي & & \\
\hline & & & Pa/APT & PYq & $A \Delta P \& \mid 8 \Delta 1$ & Sل & & \\
\hline \multirow{3}{*}{ Mf. } & \multirow{3}{*}{$.1 \cdot . r$} & \multirow{3}{*}{ Y/.. } & $15 / 9.9$ & $r$ & rav/Ter & بين كروهي & \multirow{3}{*}{ شستوشو } & \\
\hline & & & E/fDQ & req & MTAIMTE & درون كروهي & & \\
\hline & & & $19 / \mathrm{KgF}$ & r99 & TVENQM & كل & & \\
\hline \multirow{3}{*}{ - Iro } & \multirow{3}{*}{$+1+\infty$} & \multirow{3}{*}{ 1/199 } & ITAT/QIY & r. & TVEIV/TAS & بين كروهى & \multirow{3}{*}{ كل نمره ADL } & \\
\hline & & & $891 / 111$ & raq & TETVAS/THD & درونكّوهى & & \\
\hline & & & $1910 /+r$ & prq & $r+\mid f+r / \Delta q 1$ & كل & & \\
\hline \multirow{3}{*}{ * $+r \alpha$} & \multirow{3}{*}{$+1++1$} & & $114 r / 9 r^{m}$ & 1 & $114 r / 9 W^{\infty}$ & بين كروهى & & \\
\hline & & $I T / T+4$ & 9 qTVAr & rtA & $F+$ ITA/ART & درون كروهى & فغاليت & \\
\hline & & & IATV/VTA & rrq & Firequis & كل & & \\
\hline & & & qRT/FeV & 1 & qfT/FqY & بين كروهي & & \\
\hline$\cdot 1+m$ & $\%$ & If/Arq & $9 \Psi / \Delta \Delta \&$ & PrA & $r r+r /++1$ & درون كروهى & اعثقادى & \\
\hline & & & $1.091 \cdot \mathrm{m}$ & prq & rAlff/feV & كل & & \\
\hline & & & AIV/aV. & 1 & $A I V / \Delta V$. & يين كروهي & & \\
\hline.$/ . \Delta$ & $1 \ldots$ & MT/PE. & $r e / p+1$ & PrA & 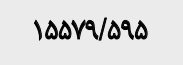 & نرون كروهى & شخصى & \\
\hline & & & 1هr/qVI & prq & IFTaV/IED & كل & & سطح \\
\hline & & & |มู/EV & 1 & MA/IEV & بين كروهى & & تحصيلات \\
\hline . $1 . r T$ & $.1 .0 Y$ & $V \& M$ & IVATY & FrA & ATAV/FAF & رون كروهى & ظريف & \\
\hline & & & r.NEAY & PYq & AAFE/EAI & كل & & \\
\hline & & & PNEAI & 1 & PNPEI & بين كروهى & & \\
\hline $.1 \cdot 11$ & $.1+.1$ & VIITY & g/ATD & ral & $M T \cdot / M I Q$ & درون كروهي & شستوشو & \\
\hline & & & $\Delta \mathrm{Q} / \Delta 18$ & m9q & TVEN99Y & كل & & \\
\hline & & & Ireqp/r+A & 1 & Ireaf/r+1 & يين كروهى & & \\
\hline .1 .80 &... & $r \cdot / r r r$ & ENT/MIA & PTA & TAW.QVAT & درون كروهي & كل نماه & \\
\hline & & & IfTEe/are & ruq & $r \cdot \mid f-r / \Delta q 1$ & كل & & \\
\hline
\end{tabular}




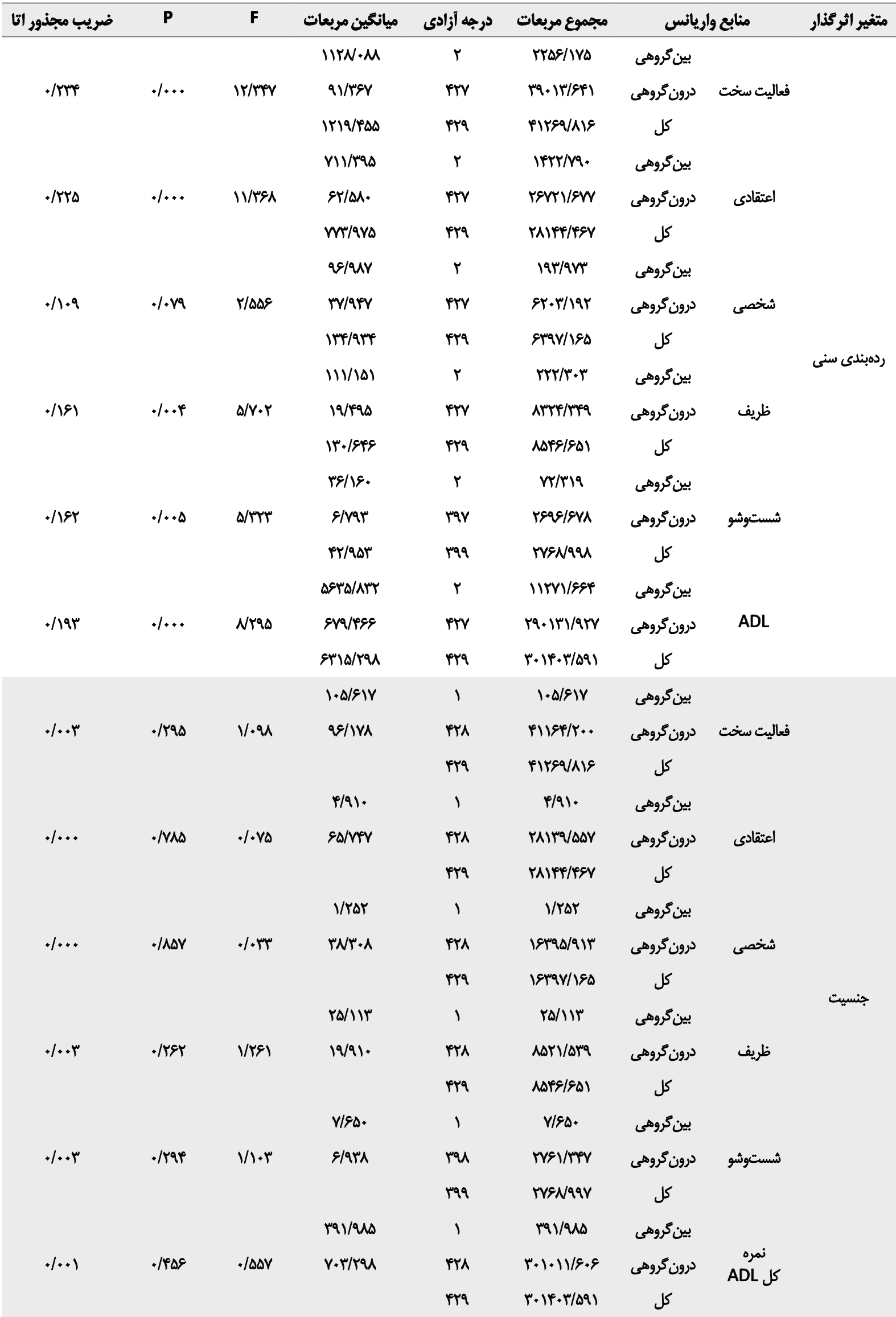




\begin{tabular}{|c|c|c|c|c|c|c|c|c|}
\hline ضريب مجذور اتا & $\mathbf{P}$ & $\mathbf{F}$ & مياتكين مربعات & درجه آزادى & مجموع مربعات & \multicolumn{2}{|c|}{ منابع وارياتس } & \multirow{4}{*}{ تثغير اثركذار } \\
\hline \multirow{3}{*}{$+1+1 r$} & \multirow{3}{*}{.$/ \%$} & \multirow{3}{*}{ rigra } & \multirow{3}{*}{$\begin{array}{l}r \Delta \Delta / Y P \Delta \\
Q \Delta / F \Delta \Gamma\end{array}$} & $r$ & $\Delta 11 / p q$. & بين كروهى & & \\
\hline & & & & prV & F.VDNHTE & درون كروهى & فعاليت سخت & \\
\hline & & & & prq & firsaluis & كل & & \\
\hline \multirow{3}{*}{$+1+1 \mathrm{~V}$} & \multirow{3}{*}{$+1+4 q$} & \multirow{3}{*}{ M/OAY } & \multirow{3}{*}{ SF/ATA } & $r$ & $\mid x q / \times q)$ & بين كروهى & \multirow{3}{*}{ اعتقادى } & \\
\hline & & & & rrV & TWEA+1+W & درون كروهى & & \\
\hline & & & & rrq & rAlfE/TEY & كل & & \\
\hline \multirow{3}{*}{$+1+\infty 1$} & \multirow{3}{*}{. Mes } & - /raf & W/TAE & $r$ & MT/AIT & بين كروهى & \multirow{3}{*}{ شخصى } & \\
\hline & & & TNMPA & PrV & ierrefear & درون كروهى & & \\
\hline & & & & prq & Ierqu/Ies & كل & & \\
\hline \multirow{3}{*}{$.1 .+1$} & \multirow{3}{*}{ - 1498} & $+M 1$ & $r / r+1$ & r & Plf.r & يين كروهى & \multirow{3}{*}{ ظريف } & \\
\hline & & & $r+1+\infty$ & prr & ADFT/TA. & درون كروهي & & \\
\hline & & & & prq & ADFE/EAI & كل & & \\
\hline \multirow{3}{*}{$.1 . .1$} & \multirow{3}{*}{.$/ 9}$. & $+11 \cdot 0$ & $\cdot M r$ & $r$ & I IFET & يين كروهي & \multirow{3}{*}{ شستوشو } & \\
\hline & & & & rav & TVEV/ATA & درون كروهى & & \\
\hline & & & & r9q & TVENQY & كل & & \\
\hline \multirow{3}{*}{$+1+\cdot V$} & \multirow[t]{3}{*}{ - Mre } & 1/Fia & $997 / 11$ eq & r & 144/FqV & يين كروهي & \multirow{3}{*}{$\begin{array}{c}\text { نمره كل } \\
\text { ADLL }\end{array}$} & \\
\hline & & & $V \cdot 1 / M 18$ & erv & rqq7iq/TqF & درون كروهي & & \\
\hline & & & & prq & $r+1 f+r / \Delta q 1$ & كل & & \\
\hline
\end{tabular}

L

فعاليت اعتقادى و اجتماعى و فعاليت ظريف با حمايت اجتماعى

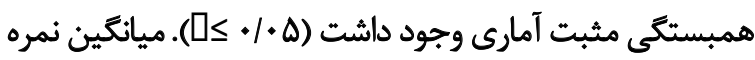

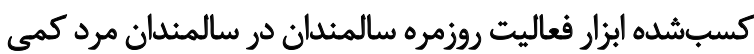

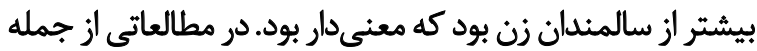

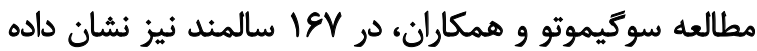

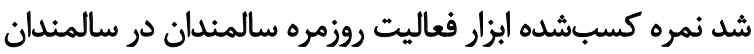

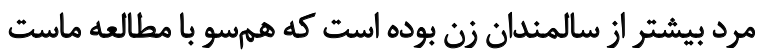

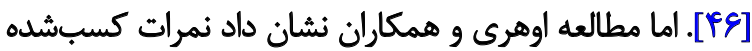

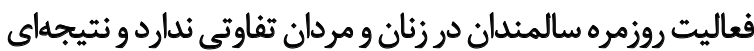

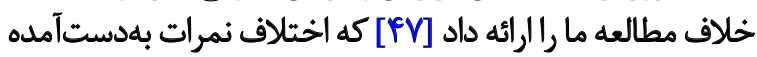
مىتواند ناشى از تفاوت در محدوده حركتى مفاصل و

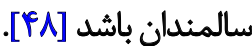

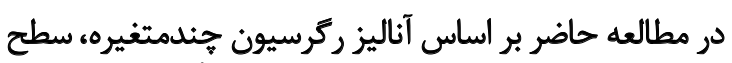

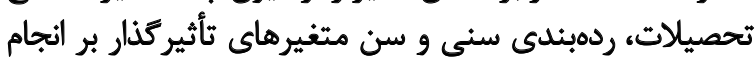

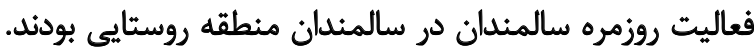

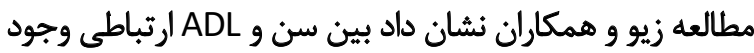

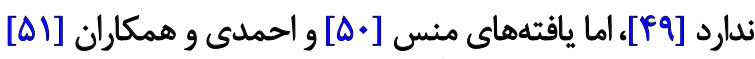
برخلاف آن نشان دادند تأثير عامل سن، معنى إمداف

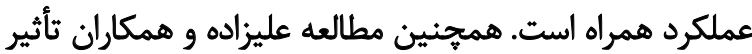
سن بر سطح ADL را نشان داد كه همرسو با نتايج ماست [AT)
Adjusted R Square $=\cdot \pi \cdot r$.PS $/ 1 \cdot \Delta$

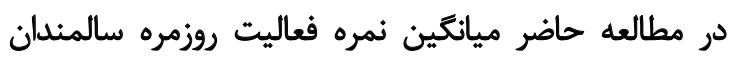

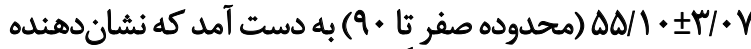

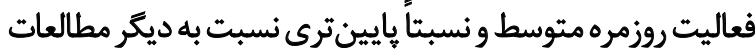

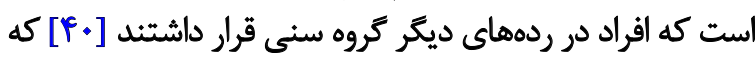

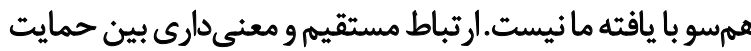

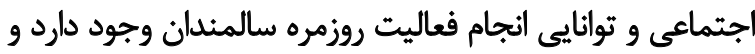

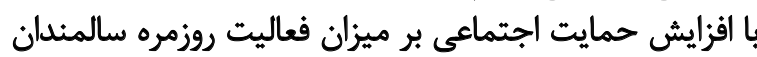

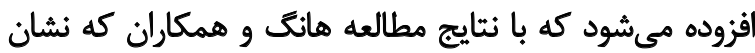

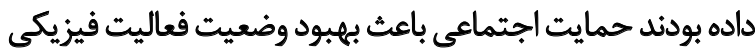

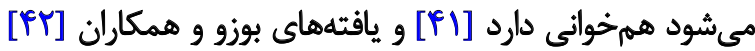

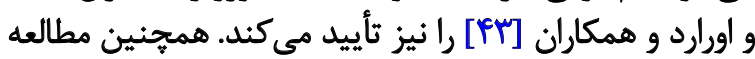

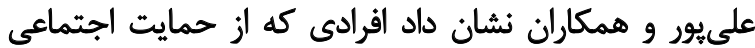

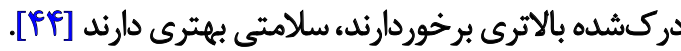
در اين بثروهش ميانكين فعاليت روزمره سالمندان،

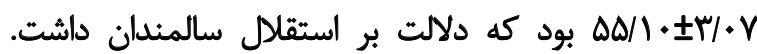

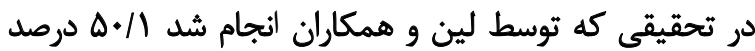

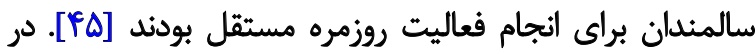

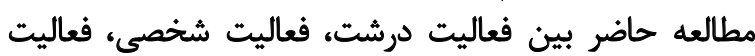

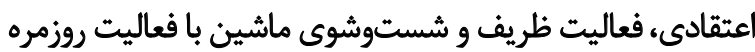

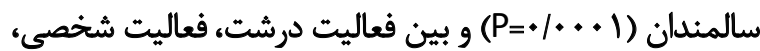


جدول ب. نتايج تحليل واريائس جندمتغيره اثر شاخص حمايت اجتماعى بر سطح ADL سالمندان روستايى

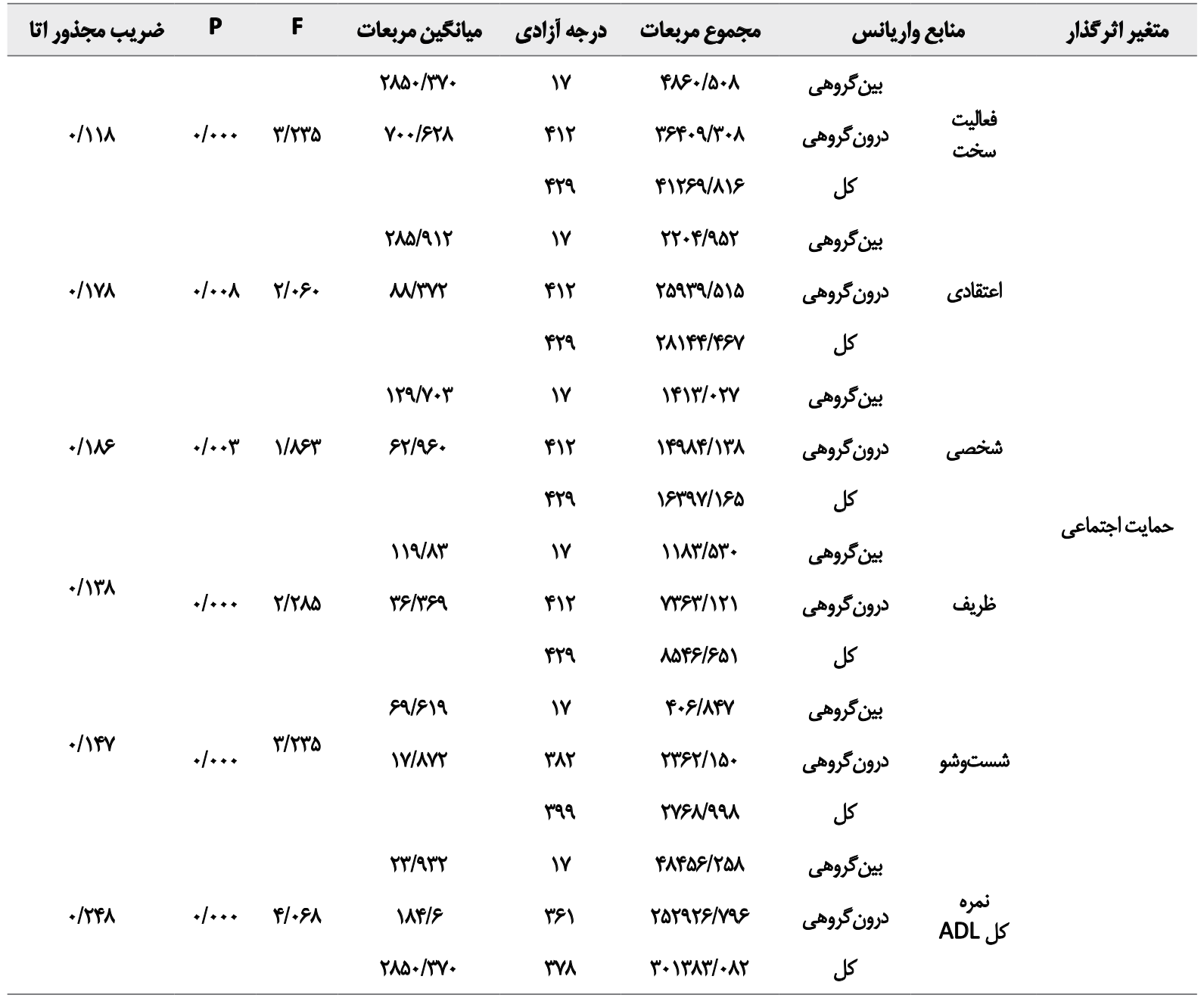

L

Adjusted R Square $=\cdot / \mathrm{MA}, \mathrm{P} \leq \cdot / \cdot \Delta$.

جسم آنها مىشود و زندكى آنها را تحت تأثير قرار ميدهد.

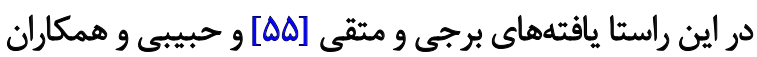

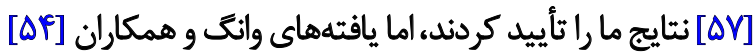

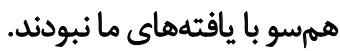

\section{نتيجهيرى نهايى}

نتايج مطالعه حاضر نشان داد بيشتر سالمندان از نظر فعاليت

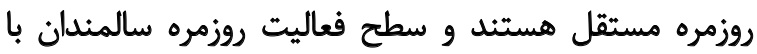

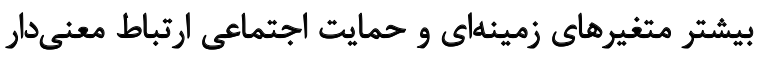

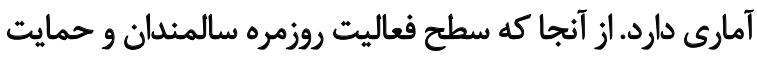

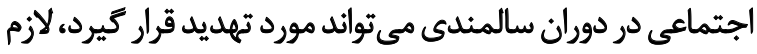

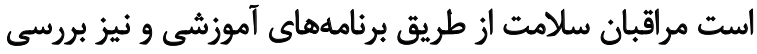

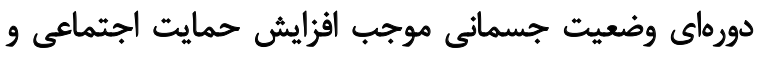
حفظ و ارتقاى زندگى سالم و فعال براى آنها شوند آندايت از مشكلات و محدوديتهاى مطالعه حاضر اين بود كه نتايج
واقعيت اين است كه با افزايش سن كنشهائ زيستشناختى

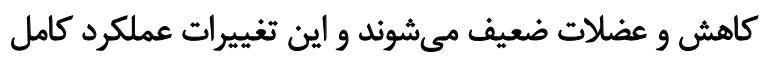

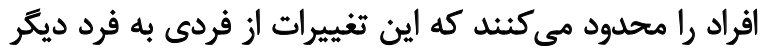

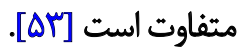
يافتههاى ما نشان دادند وضعيت تأهل مؤلفه اثر كذارى بر سطح

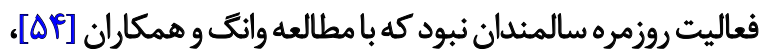

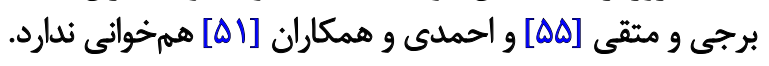

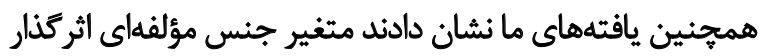

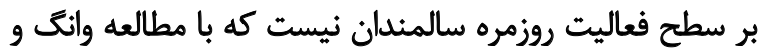

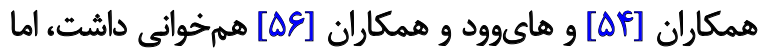

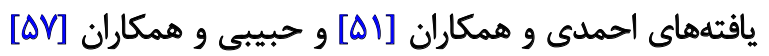

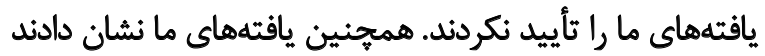

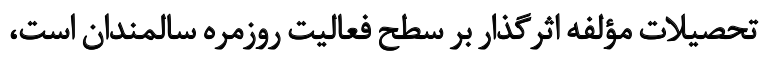

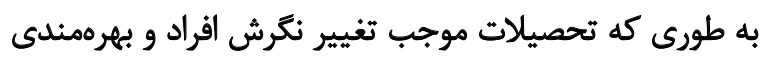

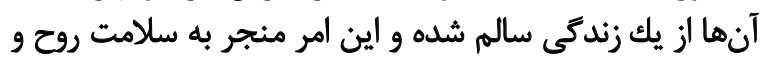




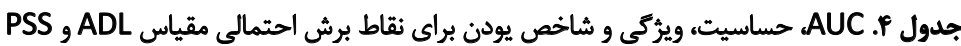

\begin{tabular}{|c|c|c|c|c|c|c|c|c|c|c|}
\hline \multirow{2}{*}{ شاخص يودن } & \multirow{2}{*}{ ويثوكى } & \multirow{2}{*}{ حساسيت } & \multirow{2}{*}{ نقط نبش } & \multirow{2}{*}{ نمرات } & \multirow{2}{*}{ مياتكين||نحرافمعيار } & \multicolumn{2}{|c|}{ فاصله اطمينان ه9 درصد } & \multirow{2}{*}{ AUC } & \multirow{2}{*}{\multicolumn{2}{|c|}{ مثغيرها }} \\
\hline & & & & & & كران هايين & كران بالا & & & \\
\hline - MAr &.$/ 914$ & - $|A Y|$ & $M / \Delta$ & PQt 9 & $|p| \cdot q \pm q / M \mid$ & .1980 & - Neq & . MIV & سخت & \\
\hline .1019 & - /Arq & •/VAI & $1 \% / \Delta$ & $r \cdot t A$ & $1 \% / 1 \cdot \pm V / \Delta$ & . Iaty & $y+\ldots$ & / ANTf & اعتقادى & \\
\hline .1811 & .19 .8 & $\cdot N \cdot \Delta$ & 18 & Tatr & $|\&| \Delta| \pm \&| \cdot 1$ & $\cdot \mu r \cdot V$ & $1 / \ldots$ &.$M T$ & شخصى & روزاليت \\
\hline .1024 & $\cdot / 9 \cdot V$ &.$|89|$ & $V / 19$ & $r+t r$ & $V / \mu q \pm V / r^{\mu}$ & IANE & $y / \ldots$ & $\cdot / \lambda+\lambda$ & ظريف & \\
\hline .108 & . IATY & . MTr & $r / \Delta \Delta$ & 1. Er & $r / \Lambda \cdot \pm r / \Lambda$. & $\cdot 18 \cdot f^{4}$ & $y+\ldots$ & $. / A \mid S$ & شستوشو & \\
\hline . MT &.$/ 9.1$ & - $/ A M$ & $P 1 / \Delta$ & 10. E. & $\Delta \Delta / / \cdot \pm r / \cdot V$ &.$/ 199$ & $1 / \ldots$ &.$/ 901$ & رهره & \\
\hline . MIT & $+(199)$ & . $|A M|$ & $1 \& / T V$ & $r+t$ & $1 \% / \Delta \Delta \pm \Delta / 19$ &.$M F$ & $\cdot / 119$ &. WE & إجتماعي & حمريت \\
\hline
\end{tabular}

جدول ه. تأثير متغير كروههاى سنى بر سطح ADL سالمندان روستايى

\begin{tabular}{|c|c|c|c|c|c|c|}
\hline نمره كل ADL & شستوثشو & ظريف & شخصى & اعتقادى & سخت & تروه سنى \\
\hline$\Delta Q / r \backslash \pm T \Delta / A \Delta$ & r/fitr/eg & $N \cdot A \pm F / F$ & $\mid V / I r \pm \Delta / \Delta q$ & $I V / I q \pm V / \Delta A$ & $10 / 4 \& \pm Q / \Delta q$ & 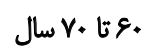 \\
\hline 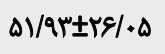 & $Y / N \& \pm Y / \Delta S$ & $V / \cdot q \pm f / r$. & $\mid E / R \Delta \pm \& / T \Delta$ & $\mid f / \& q \pm N M$ & $11 / \varepsilon \Delta \pm N \cdot V$ & J A \\
\hline HI/TEITN-1 & $1 / 9+ \pm r / r \Delta$ & $\Delta / \Delta r \pm \Delta / \omega)$ & IF/ATEVINE & $1.189 \pm N G F$ & NTFEV/NE & •1 سال و بيشتر \\
\hline$\Delta \Delta / \& \cdot \pm r g(\Delta)$ & $r / I r \pm r / g r$ & V/ALEff/Ee & $18 / M^{\prime} \pm s / 11$ & $|\triangle / 9 Y \pm N|$. & $|r / V| \pm 9 / \lambda \mid$ & كل \\
\hline
\end{tabular}

$\stackrel{2}{2}$

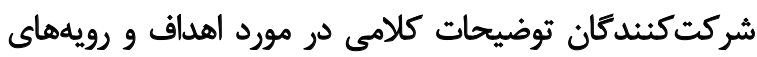

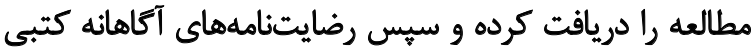

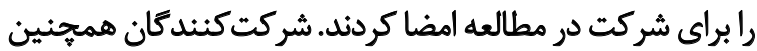

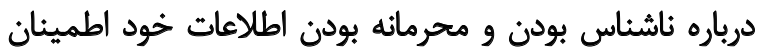

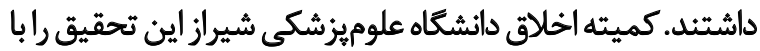

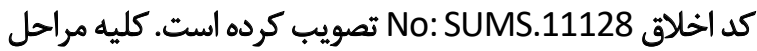

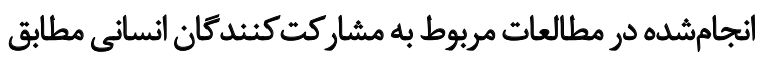

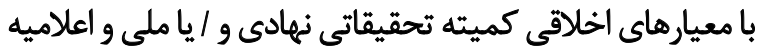

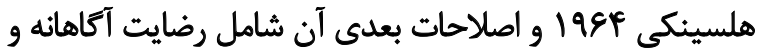
محرمانه بودن كليه اطلاعات شخصى است. مامي مالى اين ثرؤشش با حمايت و تأييد معاونت يُروهشى دانشكده بهداشت دانشكاه علوميزشكى شيراز انجام ترفته است.
اين مطالعه ممكن است قابل تعميم به ساير نقاط نباشد. يُيشنهاد

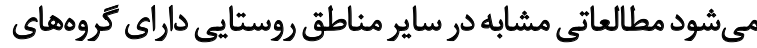

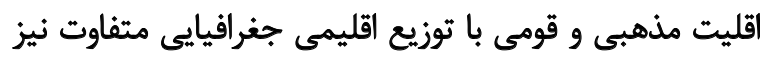

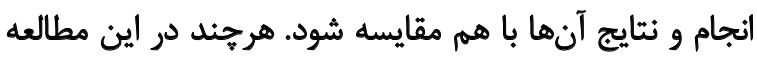

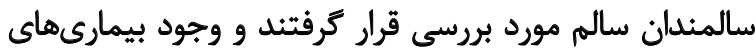

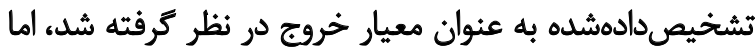

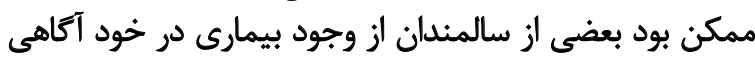

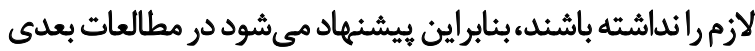

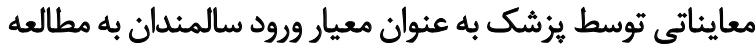

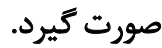

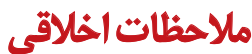

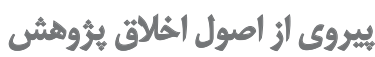
مطالعه حاضر از اصول اخلاق يُووهش بيروى مى كند. همه 


$$
\text { مشاركت نويسندكان }
$$

طراحى مطالعه، انجام مصاحبه: فاطمه جوكار؛ نظارت و نوشتن

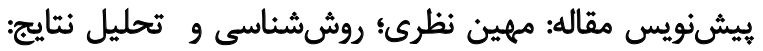

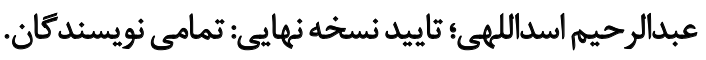

$$
\text { تعارض مناقع }
$$

نويسندكان اظهار كردند كه اين مقاله تعارض منافع ندارد. 


\section{References}

[1] World Health Organization, US National Institute of Aging, editors. Global health and aging [Internet]. 2011 [Updated 2011 October]. Available from: https:/ / www.who.int/ageing/publications/global_health/en/

[2] World Health Organization. World report on ageing and health [Internet]. 2015 [Updated 2015]. Available from: https:/ /www. who.int/ageing/publications/world-report-2015/en/

[3] Ministry of Health and Medical Education. [Training guide integrated care comprehencive elderly special medicine (Persian)] [Internet]. 2009 [Updated 2009]. Available from: https://phc. mui.ac.ir/sites/phc.mui.ac.ir/files/aein-name/gheire $\% 20$ pezeshk $\% 20 \% 281 \% 29$.pdf

[4] Ghaffari F, Dehghan-Nayeri N, Navabi N, Seylani Kh. Evaluation of the Master's curriculum for elderly nursing: A qualitative study. Clinical Interventions in Aging. 2016; 11:1333-42. [DOI:10.2147/CIA.S109004] [PMID] [PMCID]

[5] Nourbakhsh SF, Fadayevatan R, Alizadeh-Khoei M, Sharifi F. [Determining the status of Activity of Daily Living (ADL) and Instrumental Activity of Daily Living (IADL) in healthy and cognitive impaired elderlies (Persian)]. Jorjani Biomedicine Journal. 2018; 5(2):63-77. http://goums.ac.ir/jorjanijournal/article1-561-en.html

[6] Seo K, Cho M. The effects of a balloon-blowing exercise in a $90 / 90$ bridge position using a ball on the pulmonary function of females in their twenties. Journal of Physical Therapy Science. 2018; 30(10):1267-70. [DOI:10.1589/jpts.30.1267] [PMID] [PMCID]

[7] Heydari H, Shahsavari H, Hazini A, Nikbakht Nasrabadi AR. Exploring the barriers of home care services in Iran: A qualitative study. Scientifica. 2016; 2016:2056470. [DOI:10.1155/2016/2056470] [PMID] [PMCID]

[8] Osakwe ZT, Larson E, Agrawal M, Shang J. Assessment of activity of daily living among older adult patients in home healthcare and skilled nursing facilities: An integrative review. Home Healthcare Now. 2017; 35(5):258-67. [DOI:10.1097/ NHH.0000000000000537] [PMID] [PMCID]

[9] Chen Sh, Zheng J, Chen C, Xing Y, Cui Y, Ding Y, et al. Unmet needs of activities of daily living among a community-based sample of disabled elderly people in Eastern China: A cross-sectional study. BMC Geriatrics. 2018; 18(1):160. [DOI:10.1186/s12877018-0856-6] [PMID] [PMCID]

[10] Chan CS, Slaughter SE, Jones CA, Wagg AS, editors. Greater independence in activities of daily living is associated with higher health-related quality of life scores in nursing home residents with Dementia. Healthcare. 2015; 3(3):503-18. [DOI:10.3390/ healthcare3030503] [PMID] [PMCID]

[11] Mlinac ME, Feng MC. Assessment of activities of daily living, self-care, and independence. Archives of Clinical Neuropsychology. 2016; 31(6):506-16. [DOI:10.1093/arclin/acw049] [PMID]

[12] Henry-Sánchez JT, Kurichi JE, Xie D, Pan Q, Stineman MG. Do elderly people at more severe activity of daily living limitation stages fall more? American Journal of Physical Medicine \& Rehabilitation. 2012; 91(7):601-10. [DOI:10.1097/ PHM.0b013e31825596af] [PMID] [PMCID]

[13] Hillcoat-Nallétamby S. The meaning of "independence" for older people in different residential settings. The Journals of Gerontology: Series B. 2014; 69(3):419-30. [DOI:10.1093/geronb/ gbu008] [PMID]
[14] Udayshankar PM, Parameaswari PJ. Healthy and active ageing. World Applied Sciences Journal. 2014; 30(7):927-8 [DOI:10.5829/idosi.wasj.2014.30.07.82124]

[15] Lagiewka K. European innovation partnership on active and healthy ageing: Triggers of setting the headline target of 2 additional healthy life years at birth at EU average by 2020. Archives of Public Health. 2012; 70(1):23 [DOI:10.1186/0778-7367-70-23] [PMID] [PMCID]

[16] Moran M, Van Cauwenberg J, Hercky-Linnewiel R, Cerin E, Deforche B, Plaut P. Understanding the relationships between the physical environment and physical activity in older adults: A systematic review of qualitative studies. International Journal of Behavioral Nutrition and Physical Activity. 2014; 11:79. [DOI:10.1186/1479-5868-11-79] [PMID] [PMCID]

[17] Caspersen CJ, Powell KE, Christenson GM. Physical activity, exercise, and physical fitness: Definitions and distinctions for health-related research. Public Health Reports. 1985; 100(2):126-31. [PMID] [PMCID]

[18] Bauer GR. Incorporating intersectionality theory into population health research methodology: Challenges and the potential to advance health equity. Social Science \& Medicine. 2014; 110:10-7. [DOI:10.1016/j.socscimed.2014.03.022 [PMID]

[19] Ashford S, Edmunds J, French DP. What is the best way to change self-efficacy to promote lifestyle and recreational physical activity? A systematic review with meta-analysis. British Journal of Health Psychology. 2010; 15(2):265-88. [DOI:10.1348/135910709X461752] [PMID]

[20] Gill TM, Desai MM, Gahbauer EA, Holford TR, Williams CS. Restricted activity among community-living older persons: Incidence, precipitants, and health care utilization. Annals of Internal Medicine. 2001; 135(5):313-2 [DOI:10.7326/0003-4819-135-5-200109040-00007] [PMID]

[21] Daffner KR. Promoting successful cognitive aging: A comprehensive review. Journal of Alzheimer's Disease. 2010; 19(4):1101-22. [DOI:10.3233/JAD-2010-1306] [PMID] [PMCID]

[22] Rashedi V, Gharib M, Rezaei M, Yazdani AA. [Social support and anxiety in the elderly of Hamedan, Iran (Persian)]. Archives of Rehabilitation. 2013; 14(2):110-5. http://rehabilitationj.uswr.ac.ir/article-1-1210-en.html

[23] Kwong EWY, Kwan AYH. Stress-management methods of the community-dwelling elderly in Hong Kong: Implications for tailoring a stress-reduction program. Geriatric Nursing. 2004; 25(2):102-6. [DOI:10.1016/j.gerinurse.2004.02.007] [PMID]

[24] Sallis JF, Bowles HR, Bauman A, Ainsworth BE, Bull FC, Craig CL, et al. Neighborhood environments and physical activity among adults in 11 countries. American Journal of Preventive Medicine. 2009; 36(6):484-90. [DOI:10.1016/j. amepre.2009.01.031] [PMID]

[25] Abbey A, Abramis DJ, Caplan RD. Effects of different sources of social support and social conflict on emotional well-being. Basic and Applied Social Psychology. 1985; 6(2):111-29. [DOI:10.1207/s15324834basp0602_2]

[26] Berkman LF. The role of social relations in health promotion. Psychosomatic Medicine. 1995; 57(3):245-54 [DOI:10.1097/00006842-199505000-00006] [PMID] 
[27] Blazer DG. Social support and mortality in an elderly community population. American Journal of Epidemiology. 1982; 115(5):684-94. [DOI:10.1093/oxfordjournals.aje.a113351] [PMID]

[28] Tesch-Roemer C, Huxhold O. Social isolation and loneliness in old age [Internet]. 2019 [Updated 2020 December 28]. Available from: https://oxfordre.com/psychology/view/10.1093/acrefore/9780190236557.001.0001/ acrefore-9780190236557-e-393 fore/9780190236557.013.393] [DOI:10.1093/acre-

[29] Singh A, Misra N. Loneliness, depression and sociability in old age. Industrial Psychiatry Journal. 2009; 18(1):51-5. [DOI:10.4103/0972-6748.57861] [PMID] [PMCID]

[30] Bozo Ö, Toksabay NE, Kürüm O. Activities of daily living, depression, and social support among elderly Turkish people. The Journal of Psychology. 2009; 143(2):193-206. [DOI:10.3200/ JRLP.143.2.193-206] [PMID]

[31] You KS, Lee H. The physical, mental, and emotional health of older people who are living alone or with relatives. Archives of Psychiatric Nursing. 2006; 20(4):193-201. [DOI:10.1016/j. apnu.2005.12.008] [PMID]

[32] Bakhtiyari M, Emaminaeini M, Hatami H, Khodakarim S, Sahaf R. [Depression and perceived social support in the elderly (Persian)]. Salmand: Iranian Journal of Ageing. 2017; 12(2):192-207. [DOI:10.21859/sija-1202192]

[33] Khajavi D, Parsa Z. [The development of psychometric properties of "the elderly's activities of daily life scale" (Persian)]. Journal of Motor Learning and Movement. 2014; 6(1):91-108. [DOI:10.22059/JMLM.2014.50192]

[34] Procidano ME, Heller K. Measures of perceived social support from friends and from family: Three validation studies. American Journal of Community Psychology. 1983; 11(1):1-24. [DOI:10.1007/BF00898416] [PMID]

[35] Scott KM, Collings SCD. Gender and the association between mental disorders and disability. Journal of Affective Disorders. 2010; 125(1-3):207-12. [DOI:10.1016/j.jad.2010.06.022] [PMID] [PMCID]

[36] Karami J, Bagheri A, Vaseghi F. [The relationship between stress and social support with a tendency to addiction (Persian)]. Paper presented at: 2nd Global Conference on Psychology and Educational Sciences, Law and Social Sciences at the Beginning of the Third Millennium. 19 May 2016; Shiraz, Iran. https://www.sid.ir/fa/seminar/ViewPaper.aspx?ID=23146

[37] Nazari M, Ghasemi S, Vafaei H, Fararouei M. The perceived social support and its relationship with some of the demographic characteristics in Primigravida pregnant women. International Journal of Nursing and Midwifery. 2015; 7(9):1415. [DOI:10.5897/IJNM2015.0186]

[38] Khalili F, Sam Sh, Sharifirad GR, Hassanzadeh A, Kazemi $\mathrm{M}$. [The relationship between perceived social support and social health of elderly (Persian)]. Journal of Health System Research. 2012; 7(6):1216-25. http://hsr.mui.ac.ir/article1-293-en.html

[39] Izadi S, Khamehvar A, Aram SS, Yazdanpanah Nozari A. [Social support and quality of life of elderly people admitted to rehabilitation centers (Persian)]. Journal of Mazandaran University of Medical Sciences. 2013; 23(105):101-9. http:// jmums.mazums.ac.ir/article-1-2765-en.html
[40] Eisapareh K, Nazari M, Kaveh MH, Ghahremani L. The relationship between job stress and health literacy with the quality of work life among Iranian industrial workers: The moderating role of social support. Current Psychology. 2020; May. [DOI:10.1007/ s12144-020-00782-5]

[41] Huang X, Yang H, Wang HH, Qiu Y, Lai X, Zhou Z, et al. The association between physical activity, mental status, and social and family support with five major non-communicable chronic diseases among elderly people: A cross-sectional study of a rural population in Southern China. International Journal of Environmental Research and Public Health. 2015; 12(10):13209-23. [DOI:10.3390/ijerph121013209] [PMID] [PMCID]

[42] Bozo Ö, Guarnaccia CA. Activities of daily living, social support, and future health of older Americans. The Journal of Psychology. 2009; 144(1):1-14. [DOI:10.1080/00223980903356032] [PMID]

[43] Everard KM, Lach HW, Fisher EB, Baum MC. Relationship of activity and social support to the functional health of older adults. The Journals of Gerontology: Series B. 2000; 55(4):S208-12. [DOI:10.1093/geronb/55.4.S208] [PMID]

[44] Alipoor F, Sajadi H, Forozan A, Biglarian A. [The role of social support in elderly quality of life (Persian)]. Social Welfare Quarterly. 2009; 8(33):149-67. http://refahj.uswr.ac.ir/article1-1862-en.html

[45] Lin LP, Hsu SW, Hsia YC, Wu CL, Chu C, Lin JD. Association of early-onset dementia with activities of daily living (ADL) in middle-aged adults with intellectual disabilities: The caregiver's perspective. Research in Developmental Disabilities. 2014; 35(3):626-31. [DOI:10.1016/j.ridd.2013.12.015] [PMID]

[46] Sugimoto H, Demura Sh, Nagasawa Y. Age and gender-related differences in physical functions of the elderly following one-year regular exercise therapy. Health. 2014; 6(8):792-801. [DOI:10.4236/health.2014.68101]

[47] Ohri P, Gupta SK, Upadhyai N. A study of daily living dependency status among elderly in an urban slum area of Dehradun. Indian Journal of Community Health. 2014; 26(4):417-22. https://www.iapsmupuk.org/journal/index.php/IJCH/ article/view/450

[48] Nakazawa A, Nakamura K, Kitamura K, Yoshizawa Y. Association between activities of daily living and mortality among institutionalized elderly adults in Japan. Journal of Epidemiology. 2012; 22(6):501-7. [DOI:10.2188/jea.JE20110153] [PMID] [PMCID]

[49] Ziv N, Roitman DM, Katz N. Problem solving, sense of coherence and instrumental ADL of elderly people with depression and normal contr ol group. Occupational Therapy International. 1999; 6(4):243-56. [DOI:10.1002/oti.100]

[50] Menec VH. The relation between everyday activities and successful aging: A 6-year longitudinal study. The Journals of Gerontology. Series B. 2003; 58(2):S74-82. [DOI:10.1093/ geronb/58.2.s74] [PMID]

[51] Ahmadi B, AminiSanii N, Bani F, Bakhtari F. Predictors of Physical Activity in Older Adults in Northwest of Iran. Elderly Health Journal. 2018; 4(2):75-80. [DOI:10.18502/ehj.v4i2.260]

[52] Alizadeh M, Rahimi A, Arshinji M, Sharifi F, Arzaghi SM, Fakhrzadeh H. [Physical health status and socio-economic outcomes on elderly in Tehran metropolitan area (Persian)]. Iranian Journal of Diabetes and Metabolism. 2013; 13(1):29-37. http:// ijdld.tums.ac.ir/article-1-5089-en.html 
[53] Crow TJ. Molecular pathology of schizophrenia: More than one disease process? British Medical Journal. 1980; 280(6207):66-8. [DOI:10.1136/bmj.280.6207.66] [PMID] [PMCID]

[54] Wang D, Zheng J, Kurosawa M, Inaba Y, Kato N. Changes in Activities of Daily Living (ADL) among elderly Chinese by marital status, living arrangement, and availability of healthcare over a 3-year period. Environmental Health and Preventive Medicine. 2009; 14(2):128-41. [DOI:10.1007/s12199-008-0072-7] [PMID] [PMCID]

[55] Borji M, Motaghi M. [The relationship between physical activity, social support and Fatigue Severity of elderly Ilam in 2016 (Persian)]. Iranian Journal of Rehabilitation Research in Nursing. 2017; 3(4):50-7. [DOI:10.21859/ijrn-03047]

[56] Haywood KL, Garratt AM, Fitzpatrick R. Quality of life in older people: A structured review of generic self-assessed health instruments. Quality of Life Research. 2005; 14(7):1651-68. [DOI:10.1007/s11136-005-1743-0] [PMID]

[57] Habibi A, Savadpoor M T, Molaei B, Shamshiri M, Ghorbani M. [Survey of physical functioning and prevalence of chronic illnesses among the elderly people (Persian)]. Salmand: Iranian Journal of Ageing. 2009; 4(3):68-78. http:/ / salmandj.uswr.ac.ir/ article-1-314-en.html 
This Page Intentionally Left Blank 